\title{
Parts per Million Detection of Alcohol Vapors via Metal Organic Framework Functionalized Surface Plasmon Resonance Sensors
}

\author{
Wouter Vandezande, ${ }^{\dagger}$ Kris P. F. Janssen, ${ }^{\ddagger}$ Filip Delport, ${ }^{\S}$ Rob Ameloot, ${ }^{\dagger}$ Dirk E. De Vos, ${ }^{\dagger}$ \\ Jeroen Lammertyn, ${ }^{\S}$ and Maarten B. J. Roeffaers* ${ }^{\dagger} \dagger$ \\ ${ }^{\dagger}$ Centre for Surface Chemistry and Catalysis, Department of Microbial and Molecular Systems, University of Leuven, Celestijnenlaan \\ 200F, Post Box 2461, 3001 Heverlee, Belgium \\ ${ }^{\ddagger}$ Molecular Imaging and Photonics, Department of Chemistry, University of Leuven, Celestijnenlaan 200F, Post Box 2404, 3001 \\ Heverlee, Belgium \\ ${ }^{\S}$ Division of Mechatronics, Biostatistics and Sensors, Department of Biosystems, University of Leuven, Willem de Croylaan 42, Post \\ Box 2428, 3001 Heverlee, Belgium
}

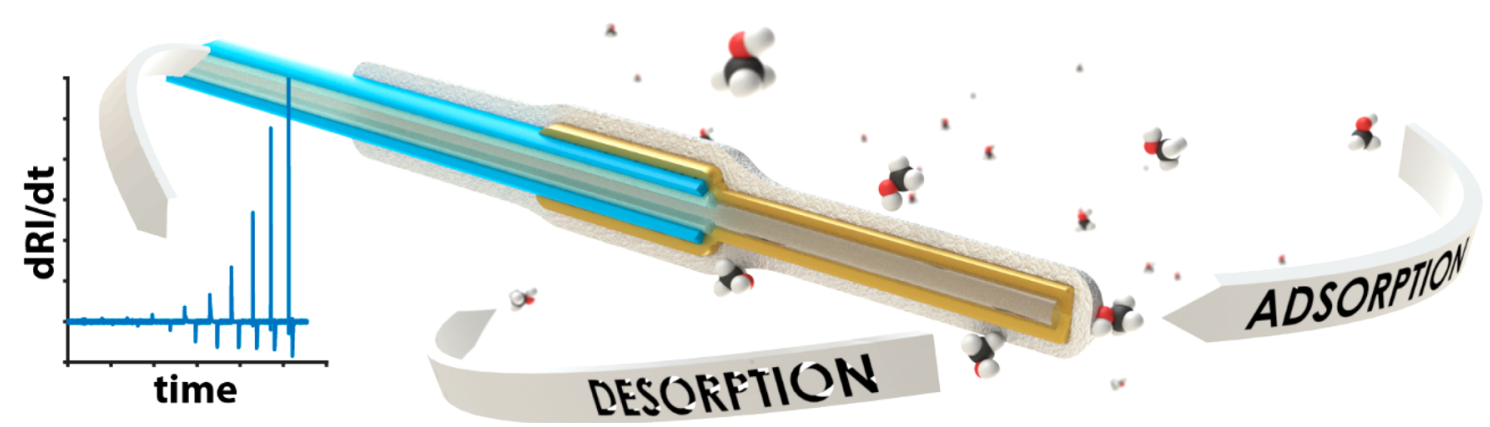

\begin{abstract}
The development of novel molecular sieves opens opportunities in the development of more sensitive analytical devices. In this paper, metal organic frameworks (MOFs), specifically ZIF-8 and ZIF-93, are grown on fiber optic based surface plasmon resonance (FO-SPR) sensors. FO-SPR has enabled sensitive sensing capabilities in biomedical settings and the addition of an MOF coating opens the way for the sensing of volatile organic compounds (VOCs) in gaseous media. FO-SPR probes were homogeneously functionalized with ZIF-8 and ZIF-93 in each case using two different precursor solutions to obtain a sequential nucleation and growth phase. The difference in MOF nucleation and growth kinetics of the two solutions was directly monitored by the FO-SPR system. The two established MOF-FO-SPR sensors were then subjected to sensing experiments with several alcohol vapors to establish their sensing capabilities. Vapors with $\mathrm{mPa}$ partial pressures, ppm concentrations, could successfully be detected, e.g., an LOD of $2.5 \mathrm{ppm}$ for methanol detection was acquired. The difference in recognition behavior of the hydrophobic ZIF-8 and more hydrophilic ZIF-93 recognition layers can be exploited to yield qualitative information regarding the vapor composition.
\end{abstract}

$\mathrm{V}$ olatile organic compounds (VOCs) have been associated with various acute and long-term health issues. Nonetheless, they are linked to virtually every form of human activity, being released from building materials, packaging, clothing but also being produced during industrial processes and transport activities. Despite their ubiquity, most VOCs are not directly detectable at relevant concentrations by our olfactory system and therefore often go unnoticed. Consequently, there is a great interest in the selective monitoring of VOCs. Currently, gas chromatography with mass spectrometry is the method of choice when quantitative and qualitative analysis is required. The high capital costs associated with this approach and the difficulty of performing real-time on-site measurements demands reliable alternatives. ${ }^{1-6}$ Hand-held devices employing photoionization detectors are nowadays used for on-site VOC analysis yielding a total measure of the VOC amount while no qualitative information on the nature of the VOC can be obtained. ${ }^{7,8}$

The difficulty in detecting VOCs lies with their typical low partial pressure, low boiling point, and low surface tension which minimizes their adherence to sensor surfaces. ${ }^{9}$ Functionalization of the sensor surface with an adsorptive layer enriches VOCs from the atmosphere at the sensor surface by several orders of magnitude enabling detection of minute amounts. The incorporation of molecular recognition into such an absorptive layer would add qualitative information. Crystalline molecular sieves might be considered good candidates for such absorptive layers. Whereas zeolites have 


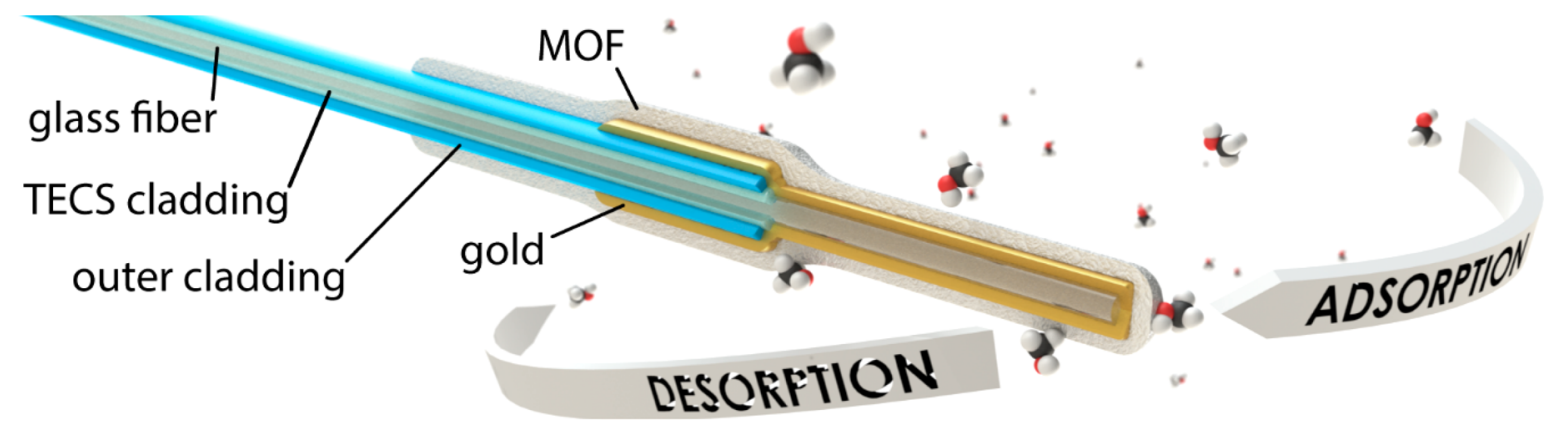

Figure 1. Schematic representation of an MOF-FO-SPR probe, not drawn to scale. The probe consists of an optical fiber with a diameter of $400 \mu \mathrm{m}$ with the inner TECS cladding and the outer protective cladding removed from the end. The lower refractive index of the TECS cladding compared with the glass core ensures the trapping of light. The $39 \mathrm{~nm}$ thick gold layer necessary for the SPR phenomenon is covered with an MOF which functions as a recognition layer. The atoms in the floating methanol molecules are oxygen (red), carbon (black), and hydrogen (white).

been extensively studied as selective sorbents, they require thermal activation to open up the pore system prior to use. ${ }^{10-12}$ Such thermal activation is incompatible with most sensor designs. In contrast, the crystalline microporosity of metalorganic frameworks (MOFs) can be evacuated under mild conditions. MOFs consist of metal ions or a metal oxide cluster interlinked by polydentate linkers into a crystalline 3D framework. Variations in both metal and organic linker have led to a very diverse group of materials being reported so far. As host-guest interactions between MOFs and VOCs can be of various nature, the MOF layer has the potential of not only serving as an adsorption layer but also as a recognition layer, providing selective adsorption toward specific analytes. ${ }^{12-16}$

Various optoelectronic approaches can be considered as a detection method in sensor development. Specifically for VOC detection surface plasmon resonance (SPR) offers some desirable properties (vide infra). In SPR, changes in refractive index (RI) of the environment around a thin metallic layer, e.g., gold, silver, copper, etc. result in a shift in the resonance wavelength. ${ }^{17-19}$ With future sensing applications in mind, a fiber optic SPR (FO-SPR) design will be used (Figure 1). In this approach, the principal FO-SPR probe consists of a stripped optical fiber coated with a layer of gold of only a few tens of nanometers thick. Light is efficiently guided through the optical fiber until it reaches the gold coated fiber tip where the surface plasmons are generated. This configuration yields flexibility in measurement design and enables physical separation of the electronics from the sensing area. The latter is important when VOCs are to be detected in hard to reach places, hazardous environments, or when there is a real risk for explosion. Compared with other SPR designs such as prismbased devices, the FO-SPR approach can easily be miniaturized as it does not contain moving components. Also, the simple design makes it perfectly suited for field use. Additionally, the active sensing area can be made replaceable and disposable, even when gold is used, as the amount of metal is minimal (5.1 $\times 10^{5} \mu \mathrm{m}^{3}$ or $9.8 \mu \mathrm{g}$ ). In comparison to silver or copper, the chemical stability of gold avoids oxidation under typical conditions. In a biological setting, the FO-SPR already has proven itself as a highly sensitive sensing system, e.g., for the identification and quantification of bacterial DNA or therapeutic drug monitoring, using antibodies and aptamers as recognizing agents. ${ }^{18-22}$

In this study, MOFs are deposited as recognition layer on top of the gold layer. More specifically two zeolitic imidazolate framework (ZIF) materials, ZIF- ${ }^{23}$ and ZIF-93, ${ }^{24}$ were explored for the detection of alcohol vapors. Alcohols were chosen as they are commonly used as solvents, fuel additives, etc. and have a strong similarity but differ sufficiently to correlate sensor output to molecule properties. Out of the many possible MOFs, ZIFs were selected to target VOCs for their small pore sizes as well as their high chemical stability. ${ }^{23-25}$ With the final design, ppm (parts per million) concentrations can repeatability be detected in a quantitative manner.

\section{EXPERIMENTAL SECTION}

Fabrication of ZIF-8-FO-SPR Sensor. FO-SPR probes were prepared and the SPR wavelengths were acquired according to the methods described by Pollet et al., ${ }^{26}$ see also Supporting Information. First, a ZIF-8 seeding layer was deposited on the golden sensing area from a fresh methanolic (methanol, Sigma-Aldrich, $\geq 99.8 \%$ ) synthesis solution (6 min) containing $21 \mathrm{mM} \mathrm{Zn}\left(\mathrm{NO}_{3}\right)_{2} \cdot 6 \mathrm{H}_{2} \mathrm{O}$ (Sigma-Aldrich, 98\%) and $198 \mathrm{mM}$ 2-methylimidazole (Sigma-Aldrich, 99\%) (mIm). ${ }^{27}$ Next, a thin ZIF-8 layer was grown from the seeding layer using a fresh methanolic synthesis solution (12 min) containing 3.67 $\mathrm{mM} \mathrm{Zn}\left(\mathrm{NO}_{3}\right)_{2} \cdot 6 \mathrm{H}_{2} \mathrm{O}, 132 \mathrm{mM}$ 2-methylimidazole $(\mathrm{mIm})$, and $99 \mathrm{mM}$ sodium formate (Sigma-Aldrich, 99\%). Between every step, the SPR probe was thoroughly washed with ethanol (Sigma-Aldrich, $\geq 99.8 \%$ ). The final SPR wavelength of the ZIF-8 coated FO-SPR sensor was $578 \pm 9 \mathrm{~nm}$ when kept in air. All steps were done at a constant temperature of $20{ }^{\circ} \mathrm{C}$. A schematic representation of the MOF-FO-SPR sensor is given in Figure 1.

Growth of ZIF-93 on an FO-SPR Probe. Similar to the deposition of ZIF-8, ZIF-93 was deposited onto the FO-SPR probe using two different synthesis mixtures. First, the nucleation of the seeding layer was promoted via a $61 \mathrm{mM}$ $\mathrm{Zn}\left(\mathrm{NO}_{3}\right)_{2} \cdot 6 \mathrm{H}_{2} \mathrm{O}$ and $225 \mathrm{mM}$ 4-methyl-5-imidazolecarboxyaldehyde (mImca) (Maybridge, 97\%) methanolic solution (6 min). Further growth of the ZIF-93 layer was done using a methanolic solution containing $3.67 \mathrm{mM} \mathrm{Zn}\left(\mathrm{NO}_{3}\right)_{2} \cdot 6 \mathrm{H}_{2} \mathrm{O}, 150$ $\mathrm{mM}$ mImca, and $99 \mathrm{mM}$ sodium formate $(6 \mathrm{~min})$. After rinsing with ethanol and air-drying, the SPR wavelength was checked and the second growth step was repeated until the SPR wavelength in air was approximately $600 \mathrm{~nm}$.

Crystallographic Characterization. Grazing incidence angle X-ray diffraction (XRD) patterns were collected on a Panalytical X'pert Pro diffractometer. XRD simulations with Rietveld refinement were performed using the software Mercury. ${ }^{28}$ All data was processed using MATLAB version $2015 b$ (The MathWorks, Inc.) except for the statistical 


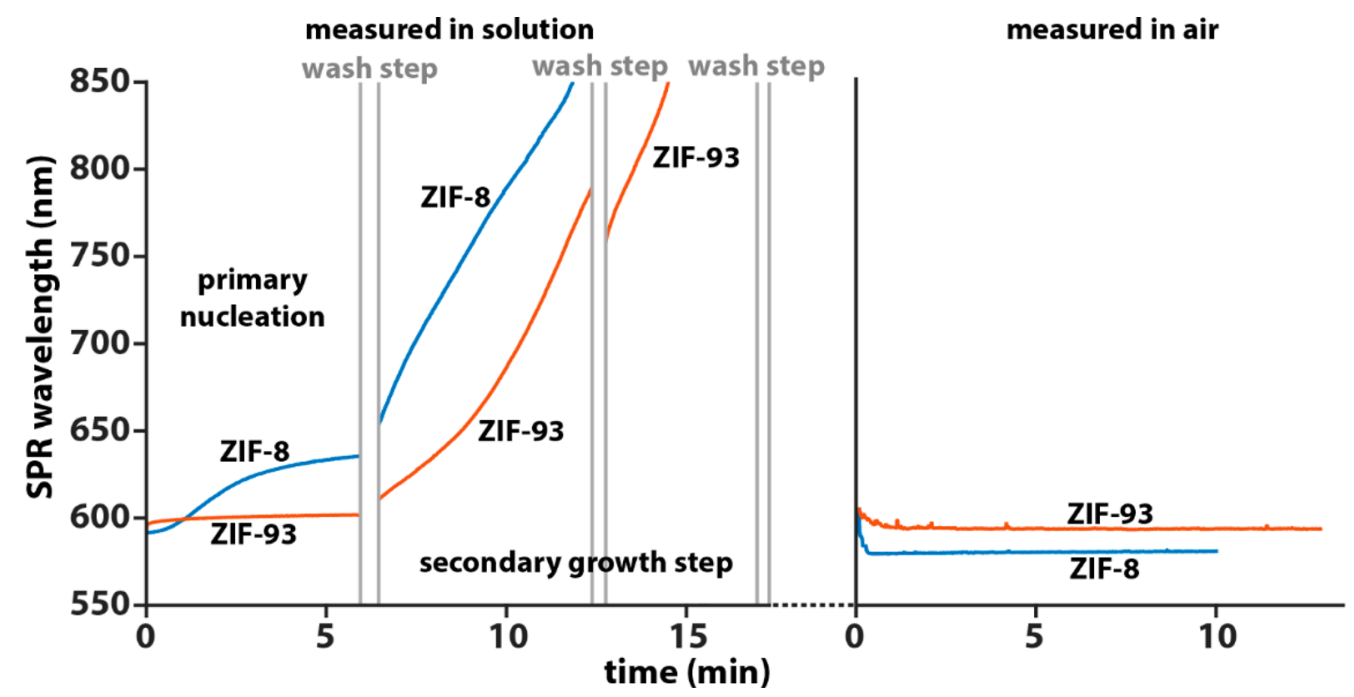

Figure 2. Deposition of ZIF-8 and ZIF-93 on the FO-SPR sensor. Two step MOF depositions are performed in precursor solutions aimed for (1) nucleation and (2) growth. After $6 \mathrm{~min}$ (ZIF-8) and $10 \mathrm{~min}$ (ZIF-93), the secondary MOF growth was stopped resulting in a stable SPR signal near $600 \mathrm{~nm}$.

calculations which were performed using Origin 8 (OriginLab Corp.).

\section{RESULTS AND DISCUSSION}

In order to produce a functional FO-SPR VOC sensor, a thin MOF layer has to be deposited on the probe surface. To achieve a homogeneous, defect free ZIF- 8 layer on the gold sensing surface, the deposition of nanosized ZIF-8 seeding crystals from the synthesis solution was optimized based on a procedure described by Lu et al. ${ }^{27}$ Here, the aim was to induce MOF nucleation rather than growth. Briefly, the FO-SPR probe was first submerged for $6 \mathrm{~min}$ in the growth solution. The deposition of the ZIF-8 seeding crystals was then followed in situ by monitoring the SPR wavelength over time (Figure 2). The nucleation step of ZIF-8 nanocrystals resulted in a fast redshift of the $50 \mathrm{~nm}$. As the SPR wavelength positively correlated with the refractive index (RI) of the local medium surrounding the SPR probe, this shift reflects the surface nucleation of ZIF-8 crystals. This observation was confirmed by the SEM images taken of the FO-SPR probe after this initial nucleation step (Figure S1A). After a wash step consisting of rinsing with ethanol (EtOH), the FO-SPR probe with ZIF-8 seeds was introduced in a growth solution optimized for crystal growth and inhibiting further nucleation by lowering the concentration of the precursors, lowering the ligand to metal ratio and the addition of formate as growth modulator; formate competes with the MOF ligands through its strong interaction with the zinc ions. ${ }^{29,30}$ Upon immersion of the FO-SPR probe into the growth solution, the SPR wavelength immediately rose fast (Figure 2) and after about 5 min the SPR wavelength exceeded the measurement range of the used spectroscopic configuration $(550-850 \mathrm{~nm})$. After $12 \mathrm{~min}$, the FO-SPR probe was removed from the solution resulting in a stable SPR wavelength around $580 \mathrm{~nm}$ in air. Although ZIF-8 has a higher refractive index than the initial growth solution, the measured SPR-wavelengths are similar because ZIF-8 only partially fills the total SPR sensing volume resulting in an effective refractive index close to this of the initial growth solution. Note that complete filling of the SPR sensing volume is not desirable as (1) the SPR signal would shift out of the spectral window and (2) mass transport in the thick ZIF-8 layer would limit sensor response. This sensor is from now on referred to as a ZIF-8-SPR sensor. Note that before ZIF-8 deposition the bare FO-SPR sensor in air $\left(n_{\text {air }}(589 \mathrm{~nm})=1.0003^{31}\right)$ showed no measurable SPR wavelength in the measured spectral range. Figure S1B shows the surface of the ZIF-8-SPR sensor probe. Clearly, the individual nanocrystals of the nucleation step were converted into a homogeneous coating. When using only the growth step without seeding layer, only a few, relatively large nanocrystals were deposited on the FO-SPR surface as opposed to the desired continuous layer (Figure S1C). To ensure that the employed protocol indeed produced the desired ZIF-8 material, the same synthesis protocols were used to generate a ZIF-8 coating on a gold coated glass slide. The acquired XRD pattern matches the reported and simulated XRD pattern of ZIF-8 (Figure 3). ${ }^{23}$ Note that the thin ZIF-8 layer only gives rise to weak Bragg reflections. For comparison, we have added the powder XRD of ZIF-8 powders obtained via a similar synthesis protocol to the Supporting Information (Figure S8).

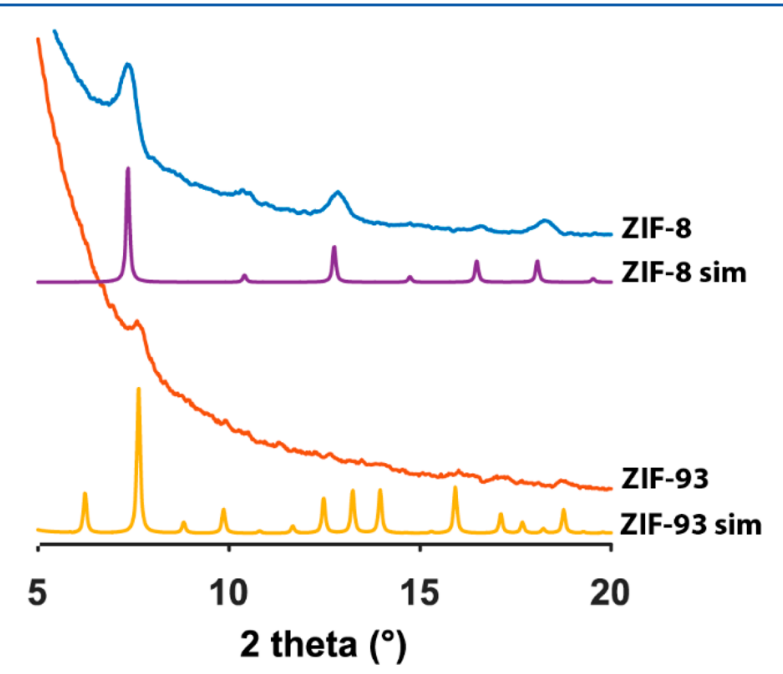

Figure 3. X-ray diffractograms of ZIF-8 and ZIF-93 surface depositions match the corresponding simulated diffractograms (sim). 
On the basis of these findings for the deposition of a stable ZIF-8 coating on the gold surface of the FO-SPR probe, an analogous procedure for the growth of ZIF-93 was developed. First, a nucleation solution was optimized resulting only in a small SPR shift due to the deposition of ZIF-93 nanocrystals on the FO-SPR probe (Figure 2). This was confirmed by SEM imaging (Figure S1D). Subsequently, a growth solution was optimized which, like the growth solution of ZIF-8, also showed a fast, immediate increase in the SPR wavelength. The secondary growth of the ZIF-93 recognition layer was checked by removing the fiber from the growth solution and checking the SPR wavelength in air. In total $10 \mathrm{~min}, 6 \mathrm{~min}$ plus 4 additional minutes in a fresh solution, were necessary to yield a stable SPR-wavelength around $600 \mathrm{~nm}$ in air. The homogeneous nature of the ZIF-93 coating was confirmed with SEM as seen in Figure S1E. Similar to ZIF-8, ZIF-93 was deposited on a gold coated glass slide and characterized by XRD. The diffractogram has enough matching peaks with the reported and simulated patterns to conclude that it is ZIF-93 (Figure 3). ${ }^{24}$ Similarly to ZIF-8, the powder XRD of ZIF-93 can be found in the Supporting Information (Figure S8).

If desired, the thickness of the final coating for both MOFs can easily be adjusted by varying the duration of the growth step. For later sensing experiments, the SPR wavelength in air was aimed to be close to $600 \mathrm{~nm}$ to make optimal use of the spectrometer range. For ZIF-8 and ZIF-93, this corresponded to a thickness of about $200 \mathrm{~nm}$, measured with SEM (Figure S2).

Having successfully prepared MOF-coated FO-SPR sensors, the applicability of these MOF coatings as selective recognition layers for alcohol vapors was evaluated. For all data presented in the next paragraphs, the SPR signal will be expressed as RI change relative to the baseline SPR signal in air. Although there is a direct relation between SPR wavelength and local RI, this is not a linear relation. Consequently, upon VOC adsorption, the magnitude of the SPR wavelength shift, with respect to the baseline, depends on the initial position of that SPR wavelength. Therefore, the SPR wavelengths were converted to the corresponding RIs by using a model of light reflection on isotropic multilayered media, as described in the literature. ${ }^{32,33}$

To ascertain the most effective operating conditions for the FO-SPR sensor, the bare FO-SPR probes were calibrated, using five different pure solvents with different RIs: 1.3270 for methanol $(\mathrm{MeOH}), 1.3330$ for water $\left(\mathrm{H}_{2} \mathrm{O}\right), 1.3604$ for $\mathrm{EtOH}$, 1.3800 for $n$-propanol ( $n$-PrOH), and 1.3913 for $n$-butanol ( $n$ $\mathrm{BuOH})$. These values correspond to the RIs at the measured SPR wavelengths and were calculated using Cauchy or Snellmeier dispersion formulas of the solvents, as found in the literature. ${ }^{34,35}$ The measurements were always performed at the temperature where the corresponding dispersion formula is valid. In the SPR model obtained from the literature, the numerical aperture (NA) and the gold layer thickness were varied. The outcome is presented in Figure S3. A good correlation $\left(R^{2}=0.98\right)$ between the experimental and fitted data was obtained for a gold layer thickness of $36 \mathrm{~nm}$ and an NA of 0.22 . The thickness of the gold layer was validated by AFM to be $39 \mathrm{~nm}$, Figure S4. Even though the NA of the SPR probe itself was $0.39,{ }^{36}$ the effective NA was determined by the smallest NA in the whole optical system which in this experiment is of the bifurcated fiber (NA 0.22). ${ }^{37}$

To study the capabilities of MOF-FO-SPR sensors, several lower alcohols were selected to generate organic vapors in dry nitrogen gas. As a first sorption experiment, four different alcohol vapors $(\mathrm{MeOH}, \mathrm{EtOH}$, isopropanol (iPrOH), and $n$ $\mathrm{BuOH})$ were introduced into the measurement chamber over both MOF-FO-SPR sensors at a relative saturation $\left(p / p_{0}\right)$ of 0.077. For direct comparison of the obtained results, the measured RI change was converted to the fractional volume occupied by the VOC inside the MOF sensing layer. This was achieved by converting the general equation for the refractive index of microporous substrates filled with a fluid analyte and/ or air: ${ }^{27}$

$$
n_{\text {eff }}^{2}=\rho_{\mathrm{a}} n_{\mathrm{a}}^{2}+\rho_{\mathrm{f}} n_{\mathrm{f}}^{2}+\left(1-\rho_{\mathrm{a}}-\rho_{\mathrm{f}}\right) n_{\mathrm{air}}{ }^{2}
$$

to the following equation:

$$
\frac{\rho_{\mathrm{a}}(t)}{\rho_{\mathrm{a}, \max }}=\frac{n_{\mathrm{eff}}{ }^{2}(t)-n_{\mathrm{eff}}{ }^{2}(0)}{\left(n_{\mathrm{a}}^{2}-{n_{\mathrm{air}}}^{2}\right)\left(1-\rho_{\mathrm{f}}\right)}
$$

where $n_{\text {eff }}(t)$ is the effective refractive index of the total MOF analyte system as measured by the SPR sensor at time $t, \rho_{a}$ and $\rho_{\mathrm{f}}$ are the volume fraction occupied by the analyte and the MOF framework $n_{\mathrm{a}}, n_{\mathrm{f}}$, and $n_{\text {air }}$ are the refractive index of the analyte, the MOF framework, and air, respectively. The maximum volume fraction which could be occupied by the analyte $\left(\rho_{\mathrm{a}, \max }\right)$ was assumed to be the volume fraction not occupied by the MOF framework $\left(1-\rho_{\mathrm{f}}\right)$, i.e., the total pore volume fraction. The outcome can be seen in Figure 4 for ZIF8 and ZIF-93. In theory, control measurements on bare FOSPR sensors should be included; however, the bare SPR sensor does not show any visible SPR activity in the measured spectral window as the RI of the surrounding vapor is too low. In theory, alcohol condensation on the SPR probe surface could result in a measurable SPR response; however, the relative saturations of the applied vapors are so low that condensation is ruled out under the measurement conditions. When the graphs of Figure 4 were compared, two significant differences between both MOF coatings were observed. First, the volume fraction occupied by the VOCs in the ZIF-93 recognition layer was about 1 order of magnitude lower than for ZIF-8 as a recognition layer for all alcohols except for $\mathrm{MeOH}$. It is known that ZIFs with carbonyl substituted ligands show a high hydrophilicity due to the strong hydrogen bonding capabilities. In contrast, ZIFs lacking polar groups on their ligand show a high hydrophobicity. ${ }^{38}$ This difference in hydrophilic-hydrophobic interactions with analytes was also at the origin of the second significant difference between both MOF-FO-SPR sensors. Whereas ZIF-8 prefers the most apolar alcohol ( $n$ $\mathrm{BuOH}$ ) over $\mathrm{EtOH}$ and $\mathrm{MeOH}$, this sequence was reversed for ZIF-93. Additionally, iPrOH adsorption in both ZIF-8 and ZIF93 was much slower to the linear alcohols. This is the result of sterically hindered diffusion in the small pores of both MOFs, which have a diameter of $3.4 \AA$ for ZIF-8 23 and 3.58 A for ZIF93. ${ }^{39}$ These findings showed that both chemical and structural interaction between the VOC and the MOF structure can be exploited as recognition properties for sensing purposes. In order to relate the VOC relative saturation $\left(p / p_{0}\right)$ to the corresponding SPR signal, the response of the MOF-FO-SPR sensors was measured at 10 different relative saturations ranging from 0.0012 to 0.25 . Each series of measurements was repeated on three distinct MOF-FO-SPR probes. Specifically, two different alcohols were tested, namely, $n-\mathrm{BuOH}$ and $\mathrm{MeOH}$. An example of the measured response using a ZIF-8SPR sensor for $n-\mathrm{BuOH}$ is shown in Figure $5 \mathrm{~A}$. At low $p / p_{0}$, it takes tens of minutes to reach steady state due to the low concentration gradient between the MOF and the surrounding 

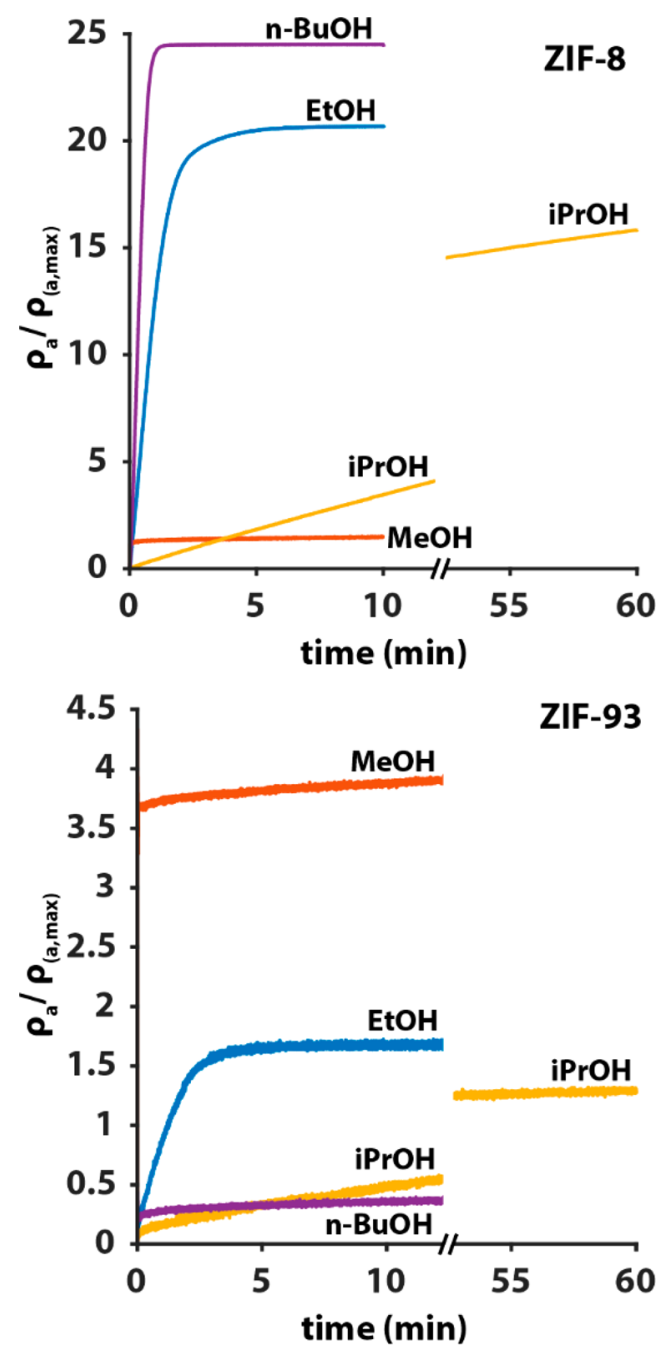

Figure 4. Volume fraction occupied by the indicated analyte from a vapor phase $\left(p / p_{0}=0.077\right)$ in reference to the total sensing volume, calculated from the SPR-response of the ZIF-8-SPR (top) and ZIF-93SPR (bottom) sensors.

air. For sensing applications, this is too long. Therefore, instead of measuring the absolute RI shift, the instantaneous change in $\mathrm{RI}$ shift $(\mathrm{dRI} / \mathrm{d} t)$ was measured enabling response in less than half a minute as the most significant changes occur fast after the introduction of the alcohol vapor. To increase the signal-tonoise ratio of the $\mathrm{dRI} / \mathrm{d} t$, a low-pass filter was used to remove the high-frequency noise (see the Supporting Information). The obtained dRI/d $t$ signal for $n-\mathrm{BuOH}$ is shown in Figure $5 \mathrm{~B}$. For $\mathrm{MeOH}$, similar graphs were obtained albeit the speed of adsorption was considerably faster.

Besides using the adsorption kinetics to determine the $p / p_{0}$ of a VOC also the desorption kinetics as well as the steady-state SPR shift were considered as an analytical measure. However, both of these require steady-state conditions to be reached, which will take too long for practical sensing purposes.

In Figure 6, $(\mathrm{dRI} / \mathrm{d} t)_{\text {peak }}$ values obtained for different $p / p_{0}$ of $n-\mathrm{BuOH}$ and $\mathrm{MeOH}$ on ZIF-8- and ZIF-93-SPR sensors are presented. In Figure S5, a similar plot is shown where $p / p_{0}$ is converted to partial pressure. The $(\mathrm{dRI} / \mathrm{d} t)_{\text {peak }}$ was the highest for the $\mathrm{MeOH}$ detection with the ZIF-93-SPR probes for almost the entire range. The carbonyl groups present on the ligands of ZIF-93 allow for the formation of hydrogen bonds,
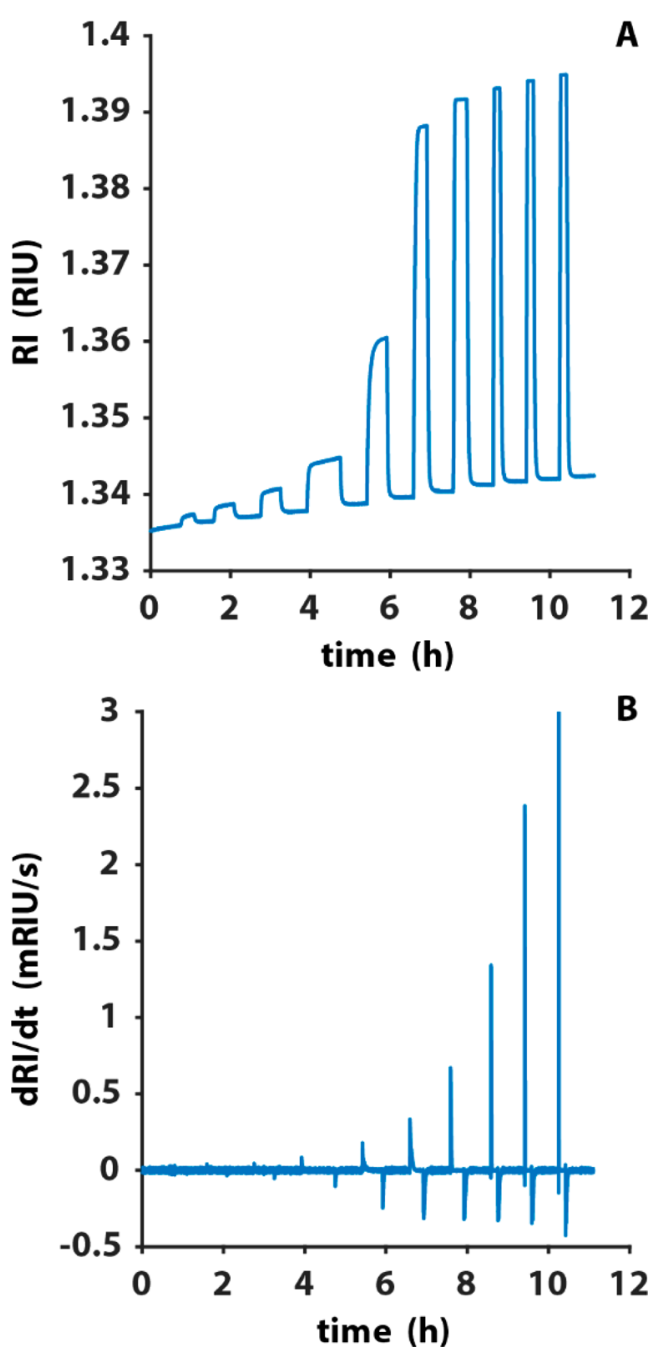

Figure 5. SPR responses of a ZIF-8-SPR sensor for 10 partial pressures of $n-\mathrm{BuOH}$, ranging from 0.0012 to 0.25 with an incremental factor of 3.16 , converted to RI (A) and differentiated to $\mathrm{dRI} / \mathrm{d} t$ followed by the application of a low-pass filter (B).

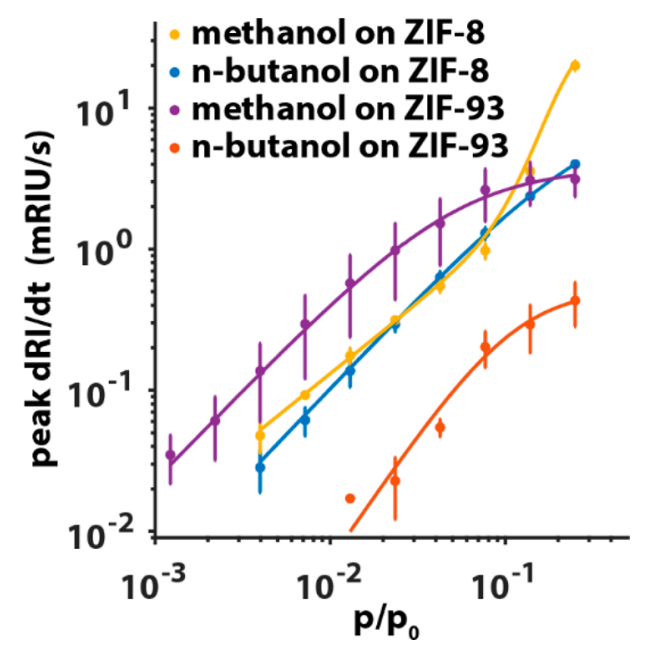

Figure 6. Calibration curves for the ZIF-8 and ZIF-93-SPR sensors for $\mathrm{MeOH}$ and $n-\mathrm{BuOH}$ vapors. The error bars indicate the standard deviation of the triplicate measurements. For all fittings, the adjusted $R^{2}$ exceeded a value of 0.97 . 
thus enabling a stronger adsorption at low $p / p_{0}$. For the same reason $n$ - $\mathrm{BuOH}$ presented the lowest $(\mathrm{dRI} / \mathrm{d} t)_{\text {peak }}$ as it is more hydrophobic. With ZIF-8, the difference between $\mathrm{MeOH}$ and $n$ - $\mathrm{BuOH}$ was small, except at the higher $p / p_{0}$ where a sudden increase in $(\mathrm{dRI} / \mathrm{d} t)_{\text {peak }}$ was observed with $\mathrm{MeOH}$. At $p / p_{0}$ of $10^{-1}$ molecular clustering of occurs due to hydrogen bond formation resulting in the sudden increase in $(\mathrm{dRI} / \mathrm{d} t)_{\text {peak }}{ }^{40}$

The use of the MOF-FO-SPR sensor for quantitative VOC sensing relied on correlating the measured $(\mathrm{dRI} / \mathrm{d} t)_{\text {peak }}$ signal to an actual $p / p_{0}$. Calibration curves were constructed by fitting the experimental data in Figure 6 with the Hill equation: ${ }^{41}$

$$
\left(\frac{\mathrm{dRI}}{\mathrm{d} t}\right)_{\text {peak }}=\left(\frac{\mathrm{dRI}}{\mathrm{d} t}\right)_{\text {peak, } \max } \cdot \frac{\left(p / p_{0}\right)^{n}}{k^{n}+\left(p / p_{0}\right)^{n}}
$$

with $(\mathrm{dRI} / \mathrm{d} t)_{\text {peak, max }}$ the maximum of $(\mathrm{dRI} / \mathrm{d} t)_{\text {peak }} n$ the Hill coefficient, and $k$ the partial pressure at half $(\mathrm{dRI} / \mathrm{d} t)_{\text {peak, max }}$. To compensate for the gate-opening effect, a linear term was added to the Hill equation:

$$
\left(\frac{\mathrm{dRI}}{\mathrm{d} t}\right)_{\text {peak }}=\left(\frac{\mathrm{dRI}}{\mathrm{d} t}\right)_{\text {peak,max }} \cdot\left(\frac{\left(p / p_{0}\right)^{n}}{k^{n}+\left(p / p_{0}\right)^{n}}+A \cdot p / p_{0}\right)
$$

The fitted parameters and their confidence limits can be found in Supporting Information in Table S1.

In Figure 7A,B, the sensitivities and selectivities of the MOFFO-SPR probes are shown and were calculated from the calibration curves with

$$
\text { sensitivity }=\frac{\mathrm{d}\left((\mathrm{dRI} / \mathrm{d} t)_{\text {peak }}\right)}{\mathrm{d}\left(p / p_{0}\right)}
$$

and

$$
\text { selectivity }\left(\frac{\mathrm{MeOH}}{\mathrm{n}-\mathrm{BuOH}}\right)=\frac{\text { sensitivity }_{\mathrm{MeOH}}}{\text { sensitivity }_{\mathrm{n}-\mathrm{BuOH}}}
$$

Above a $p / p_{0}$ of 0.1 , the sensitivity and selectivity were the highest for $\mathrm{MeOH}$ with the ZIF-8-SPR sensor. At lower $p / p_{0}$, the sensitivity and selectivity were the highest for $\mathrm{MeOH}$ on the ZIF-93-SPR sensor. This complementary behavior of these MOF-FO-SPR sensors for the sensitivity and selectivity toward $\mathrm{MeOH}$ is related to the higher affinity at lower $p / p_{0}$ for ZIF-93. While at higher $p / p_{0}$, the higher sensitivity was ensured by the molecular clustering of $\mathrm{MeOH}$ in ZIF-8 as discussed before. Almost no preference for either $\mathrm{MeOH}$ and $n-\mathrm{BuOH}$ was observed with the ZIF-8-SPR probe at low $p / p_{0}$. However, the sensitivity of the ZIF-8-SPR probe toward $\mathrm{MeOH}$ below a $p / p_{0}$ of 0.03 was near constant around $13 \mathrm{mRIU} / \mathrm{s}$. Thus, the relation between the signal of the sensor and $\mathrm{MeOH}$ partial pressure was linear, making field use very practical as no complex calibration curve is necessary for the conversions of the sensor signal to partial pressures of the analytes. As analogous adsorption kinetics is observed for $n-\mathrm{BuOH}$ and $\mathrm{MeOH}$ on ZIF-8 in literature, where the steep increase in $n$ $\mathrm{BuOH}$ adsorption was more toward lower $p / p_{0}$, a similar, desired linear relation between the ZIF-8-SPR sensor signal and $n$ - $\mathrm{BuOH} p / p_{0}$ is presumed at lower $p / p_{0}$ than measured in this work. $^{42}$ The sensitivity of the ZIF-93-sensor toward the more hydrophobic $n-\mathrm{BuOH}$ was the lowest of all and thus the selectivity of the ZIF-93-SPR sensor toward $\mathrm{MeOH}$ was at least substantial for the whole measured range.

The estimated LODs for the sensing of $\mathrm{MeOH}$ and $n-\mathrm{BuOH}$ vapors were obtained by converting the lowest detectable (dRI/
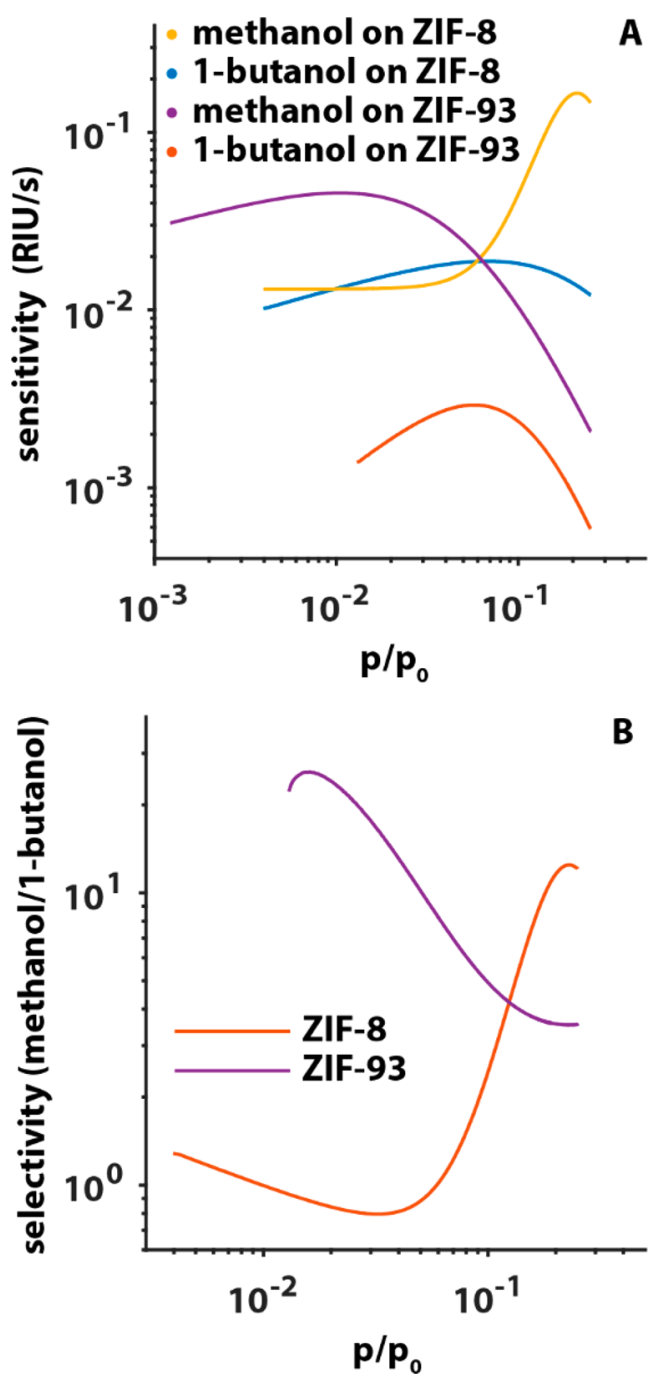

Figure 7. Curves of the sensitivity for $\mathrm{MeOH}$ and $n-\mathrm{BuOH}(\mathrm{A})$ and selectivity toward $\mathrm{MeOH}$ in respect to $n-\mathrm{BuOH}$ (B) of the ZIF-8-SPR and ZIF-93-SPR sensors. The sensors provided a complementary sensitivity and selectivity toward $\mathrm{MeOH}$. The comparable sensitivity toward $\mathrm{MeOH}$ and $n$-BuOH of the ZIF-8-SPR sensor below a $p / p_{0}$ of 0.1 makes its selectivity to almost nothing in the same $p / p_{0}$ range. The sensitivity toward $n$-BuOH of the ZIF-93-sensor was systematically the lowest.

$\mathrm{d} t$ ) peak value. This value is acquired by adding three times the standard deviation of the noise to the average of the baseline. With the established calibration curves, the LODs were then converted to $p / p_{0}$ values for both ZIF-8-SPR and ZIF-93-SPR probes. These are shown in Figure S2. The LODs of the MOFFO-SPR sensors were of the same order, $p / p_{0}$ of $10^{-5}$ to $10^{-3}$ or 10 to $1 \mathrm{ppm}$, as other compact sensing devices generally found in the literature. ${ }^{43-46}$ The lowest LOD was obtained for $\mathrm{MeOH}$ detection with the ZIF-8-SPR probe, $p / p_{0}$ of $15 \times 10^{-6}$ or $2.5 \mathrm{ppm}$, and the highest for $n-\mathrm{BuOH}$ detection with ZIF-93SPR probe, $p / p_{0}$ of $8 \times 10^{-3}$ or $73 \mathrm{ppm}$.

To test the performance of a calibrated MOF-FO-SPR sensor, an experiment was performed by applying 80 random $p / p_{0}$ of $n-\mathrm{BuOH}$ in a $16 \mathrm{~h}$ experiment. The measured SPR signal was converted to the refractive index as shown in Figure S6A. The baseline shift (vide infra) during this long-term experiment is clearly visible. While after $1 \mathrm{~h}$, the baseline drift is only $0.0063 \mathrm{RIU}$ and after $16 \mathrm{~h}$ the baseline stabilizes at a 
relative shift of 0.0307 RIU. While significant, it is not the absolute RI signal which is used to determine the applied $p / p_{0}$ values, but the low-pass filtered, the first derivative of the RI is used (Figure S6B). As the baseline drift at short times is negligible, the baseline of this derived signal is zero during the whole measurement. The $(\mathrm{dRI} / \mathrm{d} t)_{\text {peak }}$ signal is extracted and converted to the relative saturation using the established calibration curve (Figure 6). This experimentally measured and determined $p / p_{0}$ was plotted versus the applied $p / p_{0}$ (Figure 8).

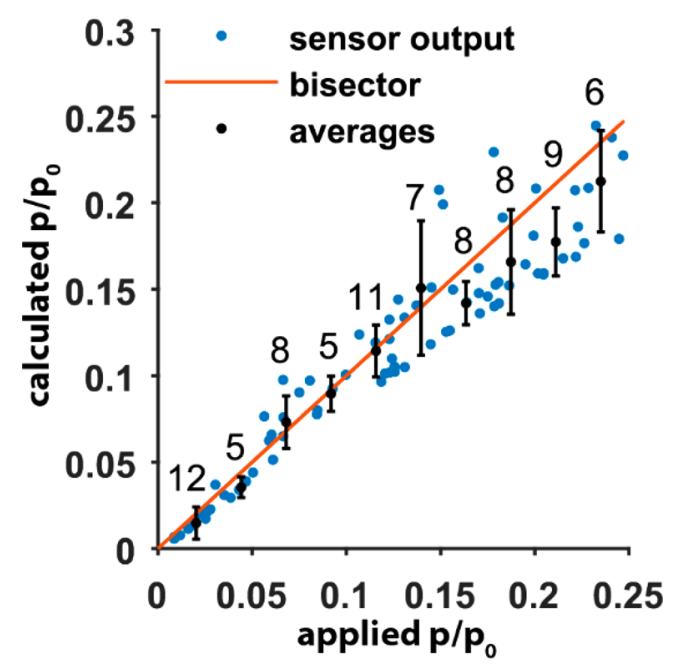

Figure 8. 80 different $n-\mathrm{BuOH}$ relative saturations measured in random order. These were further subdivided into consecutive applied $p / p_{0}$ sets. The number of data points in each set is indicated above its corresponding average. The error bars represent the standard deviation.

The acquired data points were further divided into 10 sets of consecutive, equally sized windows of applied $p / p_{0}$. The average and standard deviations are given in Table S3 and as error bars indicated in Figure 8 . For applied $p / p_{0}$ below 0.14, the response is accurate and the averages are found near the bisector. At higher relative saturations, the determined concentration underestimates the applied value. Although the precision and accuracy at higher values are still improvable, the results at lower applied $p / p_{0}$ show the reliable functioning of the MOF-FO-SPR sensor for real-life, practical applications.

During the measurements with the MOF-FO-SPR sensor, a significant baseline shift was observed after extended periods. This baseline shift is related to the gradual buildup of impurities in the sensor. Overnight exposure of a contaminated sensor to a saturated $\mathrm{MeOH}$ atmosphere and subsequent drying with $\mathrm{N}_{2}$ resulted in a full recovery of the original baseline shift. We could however not identify to origin or nature of the impurity in the used experimental setup.

\section{CONCLUSION}

In the presented work, protocols for synthesis and deposition of ZIF-8 and ZIF-93 layers under ambient conditions on an FOSPR probe were developed. The FO-SPR sensor allowed to monitor and optimize the ZIF layer deposition. The use of two different synthesis solutions was found to be crucial: one optimized for the MOF nucleation and another for further growth. The established kinetic patterns were used to then optimize two similar synthesis solutions for ZIF-93. With the similarity between ZIFs in mind, this procedure can be generalized to other ZIFs and possibly even at higher temperatures, thus, potentially establishing multiple ZIF-FOSPR sensors in an analog way.

Once the ZIF-8 and ZIF-93 coated FO-SPR sensors were established. Their applicability was tested with adsorption experiments with several alcohol vapors and monitoring the change in $\mathrm{dRI} / \mathrm{d} t$ converted from the SPR response enabling a response time under half a minute. Both the chemical and structural interactions between the alcohols and ZIFs provided saturation-dependent selectivity and sensitivity differences between both sensors with LODs to the order of $\mathrm{ppm}$. Accuracy and precision are demonstrated at low $p / p_{0}$. If the discussed baseline issue will be solved the accuracy and precision will be improved over the whole range.

These observations proved the applicability of the ZIF coated FO-SPR sensors and offers clues to the applicability to other MOFs, as they show diverse sorptive behaviors with their diverse building blocks. With applications in mind, further exploration of different MOF materials as well as expansion of the VOC pallet in the presence of water, oxygen, carbon dioxide, or other nonorganic volatiles can be included in future research.

The ability to monitor the RI with the FO-SPR setup made it possible to monitor the mass and density changes during layer formation of the ZIFs and the following sorption behavior of several alcohols on these layers. This sets the example not only to use FO-SPR as a sensing platform but also as a sensitive system to study the kinetics of material formation and sorptive behavior or even other phenomena where the RI changes.

\section{ACKNOWLEDGMENTS}

We acknowledge financial support from the Research Foundation-Flanders (FWO, Grants G.0197.11, G086114N, postdoctoral fellowship to K.P.F.J.), KU Leuven Research Fund (Grants OT/12/059, IOF/12/002, and C14/15/053), and the Belgian Federal Science Policy Office (Grant IAP-VII/05). The research leading to these results has received funding from the European Research Council under the European Union's Seventh Framework Programme (Grant FP/2007-2013)/ERC Grant Agreement No. 307523, ERC-Stg LIGHT to M.B.J.R. We thank Timothée Stassin for the grazing incidence angle XRD measurements. 


\section{REFERENCES}

(1) Dudareva, N.; Klempien, A.; Muhlemann, J. K.; Kaplan, I. New Phytol. 2013, 198 (1), 16-32.

(2) Ramírez, N.; Cuadras, A.; Rovira, E.; Borrull, F.; Marcé, R. M. Environ. Int. 2012, 39 (1), 200-209.

(3) Villeneuve, P. J.; Jerrett, M.; Brenner, D.; Su, J.; Chen, H.; McLaughlin, J. R. Am. J. Epidemiol. 2014, 179 (4), 443-451.

(4) Nurmatov, U. B.; Tagiyeva, N.; Semple, S.; Devereux, G.; Sheikh, A. Eur. Respir. Rev. 2015, 24 (135), 92-101.

(5) Lavine, B. K.; Mirjankar, N.; LeBouf, R.; Rossner, A. Microchem. J. 2012, $103(0), 37-41$.

(6) de Meij, T. G.; Larbi, I. B.; van der Schee, M. P.; Lentferink, Y. E.; Paff, T.; Terhaar sive Droste, J. S.; Mulder, C. J.; van Bodegraven, A. A.; de Boer, N. K. Int. J. Cancer 2014, 134, 1132-1138.

(7) Bello, A.; Quinn, M. M.; Perry, M. J.; Milton, D. K. Environ. Health 2010, 9 (1), 76.

(8) Morawska, L.; He, C.; Johnson, G.; Jayaratne, R.; Salthammer, T.; Wang, H.; Uhde, E.; Bostrom, T.; Modini, R.; Ayoko, G.; McGarry, P.; Wensing, M. Environ. Sci. Technol. 2009, 43 (4), 1015-1022.

(9) Salthammer, T. Indoor Air 2016, 26 (1), 25-38.

(10) Ghiaci, M.; Abbaspur, A.; Kia, R.; Seyedeyn-Azad, F. Sep. Purif. Technol. 2004, 40 (3), 217-229.

(11) Meininghaus, C. K. W.; Prins, R. Microporous Mesoporous Mater. 2000, 35, 349-365.

(12) Li, J.-R.; Kuppler, R. J.; Zhou, H.-C. Chem. Soc. Rev. 2009, 38 (5), 1477-1504.

(13) Yang, K.; Sun, Q.; Xue, F.; Lin, D. J. Hazard. Mater. 2011, 195 (0), 124-131.

(14) Huang, C.-Y.; Song, M.; Gu, Z.-Y.; Wang, H.-F.; Yan, X.-P. Environ. Sci. Technol. 2011, 45 (10), 4490-4496.

(15) Khoshaman, A. H.; Bahreyni, B. Sens. Actuators, B 2012, 162 (1), 114-119.

(16) Luebbers, M. T.; Wu, T.; Shen, L.; Masel, R. I. Langmuir 2010, 26 (13), 11319-11329.

(17) Jorgenson, R. C.; Yee, S. S. Sens. Actuators, B 1993, 12 (3), 213220.

(18) Lu, J.; Van Stappen, T.; Spasic, D.; Delport, F.; Vermeire, S.; Gils, A.; Lammertyn, J. Biosens. Bioelectron. 2016, 79, 173-179.

(19) Janssen, K. P. F.; Knez, K.; Vanysacker, L.; Schrooten, J.; Spasic, D.; Lammertyn, J. Nanotechnology 2012, 23 (23), 235503.

(20) Homola, J. Chem. Rev. 2008, 108 (2), 462-493.

(21) Wijaya, E.; Lenaerts, C.; Maricot, S.; Hastanin, J.; Habraken, S.; Vilcot, J.-P.; Boukherroub, R.; Szunerits, S. Curr. Opin. Solid State Mater. Sci. 2011, 15 (5), 208-224.

(22) Arghir, I.; Spasic, D.; Verlinden, B. E.; Delport, F.; Lammertyn, J. Sens. Actuators, B 2015, 216, 518-526.

(23) Park, K. S.; Ni, Z.; Cote, A. P.; Choi, J. Y.; Huang, R.; UribeRomo, F. J.; Chae, H. K.; O’Keeffe, M.; Yaghi, O. M. Proc. Natl. Acad. Sci. U. S. A. 2006, 103 (27), 10186-10191.

(24) Morris, W.; Leung, B.; Furukawa, H.; Yaghi, O. K.; He, N.; Hayashi, H.; Houndonougbo, Y.; Asta, M.; Laird, B. B.; Yaghi, O. M. J. Am. Chem. Soc. 2010, 132 (32), 11006-11008.

(25) Pesterfield, L. J. Chem. Educ. 2009, 86 (10), 1182.

(26) Pollet, J.; Delport, F.; Janssen, K. P. F.; Jans, K.; Maes, G.; Pfeiffer, H.; Wevers, M.; Lammertyn, J. Biosens. Bioelectron. 2009, 25 (4), 864-869.

(27) Lu, G.; Hupp, J. T. J. Am. Chem. Soc. 2010, 132 (23), 7832.

(28) Macrae, C. F.; Edgington, P. R.; McCabe, P.; Pidcock, E.; Shields, G. P.; Taylor, R.; Towler, M.; van de Streek, J. J. Appl. Crystallogr. 2006, 39 (3), 453-457.

(29) Shah, M.; Kwon, H. T.; Tran, V.; Sachdeva, S.; Jeong, H.-K. Microporous Mesoporous Mater. 2013, 165 (0), 63-69.

(30) Cravillon, J.; Schroder, C. A.; Bux, H.; Rothkirch, A.; Caro, J.; Wiebcke, M. CrystEngComm 2012, 14 (2), 492-498.

(31) Ciddor, P. E. Appl. Opt. 1996, 35 (9), 1566.

(32) Yuan, Y.; Ding, L.; Guo, Z. Sens. Actuators, B 2011, 157 (1), $240-245$.

(33) Gupta, B. D.; Sharma, A. K. Sens. Actuators, B 2005, 107 (1), $40-46$.
(34) Daimon, M.; Masumura, A. Appl. Opt. 2007, 46 (18), 38113820.

(35) Kedenburg, S.; Vieweg, M.; Gissibl, T.; Giessen, H. Opt. Mater. Express 2012, 2 (11), 1588-1611.

(36) Thorlabs Inc. 0.39 NA TECS-Clad Multimode Optical Fiber, Step Index, http://www.thorlabs.de/newgrouppage9.cfm?objectgroup_id= 6845 (accessed May 6, 2016).

(37) Ocean Optics Inc. Numerical Aperture, http://oceanoptics.com/ product-category/numerical-aperture/ (accessed May 6, 2016).

(38) Küsgens, P.; Rose, M.; Senkovska, I.; Fröde, H.; Henschel, A.; Siegle, S.; Kaskel, S. Microporous Mesoporous Mater. 2009, 120 (3), 325-330.

(39) Ray, K. G.; Olmsted, D. L.; Burton, J. M. R.; Houndonougbo, Y.; Laird, B. B.; Asta, M. Chem. Mater. 2014, 26 (13), 3976-3985.

(40) Krishna, R.; van Baten, J. M. Langmuir 2010, 26 (13), 1085410867.

(41) Gesztelyi, R.; Zsuga, J.; Kemeny-Beke, A.; Varga, B.; Juhasz, B.; Tosaki, A. Arch. Hist. Exact Sci. 2012, 66 (4), 427-438.

(42) Cousin Saint Remi, J.; Rémy, T.; Van Hunskerken, V.; van de Perre, S.; Duerinck, T.; Maes, M.; De Vos, D.; Gobechiya, E.; Kirschhock, C. E. A.; Baron, G. V.; Denayer, J. F. M. ChemSusChem 2011, 4 (8), 1074-1077.

(43) Kim, J.-S.; Yoo, H.-W.; Choi, H. O.; Jung, H.-T. Nano Lett. 2014, 14 (10), 5941-5947.

(44) Sarkar, T.; Srinives, S.; Sarkar, S.; Haddon, R. C.; Mulchandani, A. J. Phys. Chem. C 2014, 118 (3), 1602-1610.

(45) Badhulika, S.; Myung, N. V.; Mulchandani, A. Talanta 2014, 123, 109-114.

(46) Rakshit, T.; Santra, S.; Manna, I.; Ray, S. K. RSC Adv. 2014, 4 (69), 36749. 